MATEMATIKA, 2017, Volume 33, Number 1, 21-34

(C) Penerbit UTM Press. All rights reserved

\title{
Stochastic ordering constraint for ordered extremes
}

\author{
Mohd Bakri Adam \\ Department of Mathematics \& Institute of Mathematical Research \\ Universiti Putra Malaysia \\ e-mail: bakri@upm.edu.my
}

\begin{abstract}
The constraint of two ordered extreme minima random variables when one variable is consider to be stochastically smaller than the other one has been carried out in this article. The quantile functions of the probability distribution have been used to establish partial ordering between the two variables. Some extensions and generalizations are given for the stochastic ordering using the important of sign of the shape parameter.
\end{abstract}

Keywords Bivariate extreme model; generalized extreme for minima; ordered extreme stochastic ordering

2010 Mathematics Subject Classification 60G70, 62G32, 58E15.

\section{Introduction}

When comparing the size of two random variables, $X$ and $Y$, the simplest way is through their means and variances. Sometimes the mean of $X$ is smaller than that of $Y$, while the standard deviation of $Y$ is greater than than of $X$. This situation gives an unclear position for ordering $X$ and $Y$ [1]. Furthermore, sometimes these features of distributions might not exist in some cases.

A more informative method of comparing two variables is in terms of knowledge of the underlying probability distributions i.e. quantile function etc. This will establish partial ordering between the variables which is called stochastic ordering. Stochastic ordering is an ordering of random variables and their distribution functions [1]. In this article, we consider $X$ to be stochastically smaller than $Y, X<Y$, if $F_{Y}(a) \leq F_{X}(a)$ for each $a \in R$. This definition leads to a tendency for $X$ to have smaller values than $Y$. Further notes on stochastic ordering are available in [2] and references therein.

The purpose of this research is to develop bivariate extremal models and associated statistical procedures for vector observations whose components are subject to an order relationship. We develop a theorem and corollary relating to the order constraint, $Z_{x}<$ $Z_{y}$ where where $Z_{x} \sim \operatorname{GEVM}\left(\mu_{x}, \sigma_{x}, \xi_{x}\right)$ and $Z_{y} \sim \operatorname{GEVM}\left(\mu_{y}, \sigma_{y}, \xi_{y}\right)$ where GEVM is the notation for the Generalized Extreme Value for Minima distribution; and study the implications for the parameters. The location, scale and shape parameters where represent by $\mu, \sigma$ and $\xi$ respectively.

The joint structure of the extreme models for the ordered variables $\left(Z_{x}, Z_{y}\right)$ is therefore needed to extract the information for inference for the extremes data $[3,4]$. One approach to the estimation would be to fit the bivariate extreme value, BEVM, distribution to the observed pairs data, $\left(Z_{x}, Z_{y}\right)$ where

$$
\left(Z_{x}, Z_{y}\right) \sim \operatorname{BEVM}\left(\mu_{x}, \mu_{y}, \sigma_{x}, \sigma_{y}, \xi_{x}, \xi_{y}, s=1\right)
$$


where $0 \leq s \leq 1$ is the dependent scale with $s=1$ is showing the absolute independent. In this study we assume that $Z_{x}$ and $Z_{y}$ are independent of each other.

We use these results to develop an extension to the bivariate ordered extreme value cases of the smoothing techniques discussed in another article [5].

In Section 2 we introduce Theorem 1 relating to the stochastic ordering constraint, $Z_{x}<Z_{y}$, on the extreme values. Next, in the later section, we extended some conditions in Theorem 1 .

\section{Ordered extremes}

Let us assume that a block minima $Z$ of independent variables follows $\operatorname{GEVM}(\mu, \sigma, \xi)$ distribution whose parameters are to be estimated, then the distribution function for $Z$ is

$$
\operatorname{Pr}(Z<z)=H(z)= \begin{cases}1-\exp \left\{-\left[1-\xi\left(\frac{z-\mu}{\sigma}\right)\right]_{+}^{-1 / \xi}\right\} & \text { for } \quad \xi \neq 0 \\ 1-\exp \left[-\exp \left(\frac{z-\mu}{\sigma}\right)\right] & \text { for } \xi=0 .\end{cases}
$$

and the density function is

$$
h(z)= \begin{cases}\frac{1}{\sigma}\left[1-\xi\left(\frac{z-\mu}{\sigma}\right)\right]_{+}^{-1-1 / \xi} \exp \left\{-\left[1-\xi\left(\frac{z-\mu}{\sigma}\right)\right]_{+}^{-1 / \xi}\right\} & \text { for } \xi \neq 0 \\ \frac{1}{\sigma} \exp \left(\frac{z-\mu}{\sigma}\right) \exp \left[-\exp \left(\frac{z-\mu}{\sigma}\right)\right] & \text { for } \xi=0 .\end{cases}
$$

When we let $H\left(z_{p}\right)=1-p$ for $p \in[0,1]$, we get the quantiles of the GEVM distribution which has the value

$$
z_{p}=\left\{\begin{array}{lll}
\mu+\frac{\sigma}{\xi}\left[1-(-\log p)^{-\xi}\right] & \text { for } & \xi \neq 0 \\
\mu+\sigma \log (-\log p) & \text { for } & \xi=0 .
\end{array}\right.
$$

Suppose we have two GEVM distribution functions, i.e.

$$
Z_{x} \sim \operatorname{GEVM}\left(\mu_{x}, \sigma_{x}, \xi_{x}\right) \text { and } Z_{y} \sim \operatorname{GEVM}\left(\mu_{y}, \sigma_{y}, \xi_{y}\right)
$$

with quantile functions $x_{p}$ and $y_{p}$ respectively. If $Z_{x}<Z_{y}$, then Theorem 1 gives all possible conditions for the GEVM parameters.

Theorem 1 Let $Z_{x} \sim \operatorname{GEVM}\left(\mu_{x}, \sigma_{x}, \xi_{x}\right)$ and $Z_{y} \sim \operatorname{GEVM}\left(\mu_{y}, \sigma_{y}, \xi_{y}\right)$ with the respective quantile

$$
x_{p}=\mu_{x}+\frac{\sigma_{x}}{\xi_{x}}\left[1-(-\log p)^{-\xi_{x}}\right] \text { and } y_{p}=\mu_{y}+\frac{\sigma_{y}}{\xi_{y}}\left[1-(-\log p)^{-\xi_{y}}\right] \text { for } p \in[0,1] .
$$

If $Z_{x}$ and $Z_{y}$ are stochastically ordered with $Z_{x}<Z_{y}$, then the only possible conditions on the GEVM parameters are as follows:

(i) For $\xi_{y}=\xi_{x}=\xi>0$ then $\sigma_{x}>\sigma_{y}$ and $\mu_{x}+\frac{\sigma_{x}-\sigma_{y}}{\xi}<\mu_{y}$. 
(ii) For $\xi_{y}=\xi_{x}=\xi<0$ then $\sigma_{x}<\sigma_{y}$ and $\mu_{x}+\frac{\sigma_{x}-\sigma_{y}}{\xi}<\mu_{y}$.

(iii) For $\xi_{y}=\xi_{x}=\xi=0$ then $\sigma_{x}=\sigma_{y}$ and $\mu_{x}<\mu_{y}$.

(iv) For $\xi_{y}<0<\xi_{x}$ then

$$
\left.\mu_{y}+\frac{\sigma_{y}}{\xi_{y}}-\mu_{x}-\frac{\sigma_{x}}{\xi_{x}}>\sigma_{x}^{\xi_{y} /\left(\xi_{y}-\xi_{x}\right.}\right) \sigma_{y}^{-\xi_{x} /\left(\xi_{y}-\xi_{x}\right)}\left(\frac{1}{\xi_{x}}-\frac{1}{\xi_{y}}\right) .
$$

(v) For $\xi_{y}<0, \xi_{x}=0$ then

$$
\mu_{y}+\frac{\sigma_{y}}{\xi_{y}}-\mu_{x}-\frac{\sigma_{x}}{\xi_{y}}+\frac{\sigma_{x}}{\xi_{y}} \log \left(\frac{\sigma_{x}}{\sigma_{y}}\right)>0 .
$$

Essentially these conditions come from the requirement that $x_{p}<y_{p}$ for all $p$. In order to prove Theorem 1, we need to look at all 13 possible relationships between $\xi_{x}$ and $\xi_{y}$ namely:

$$
\begin{array}{lll}
\xi_{x}=\xi_{y}=0, & \xi_{x}=\xi_{y}>0, & \xi_{x}=\xi_{y}<0, \\
\xi_{x}>\xi_{y}>0, & \xi_{y}>\xi_{x}>0, & \xi_{x}<\xi_{y}<0, \\
\xi_{y}<\xi_{x}<0, & \xi_{y}<0<\xi_{x}, & \xi_{x}<0<\xi_{y}, \\
\xi_{x}=0, \xi_{y}>0, & \xi_{x}=0, \xi_{y}<0, & \\
\xi_{x}<0, \xi_{y}=0, & \xi_{x}>0, \xi_{y}=0 . &
\end{array}
$$

Proof Case 1: $\xi_{x}=\xi_{y}=\xi=0$.

Let $k_{p}^{*}=\log (-\log p)$ then $x_{p}=\mu_{x}+\sigma_{x} k_{p}^{*}$ and $y_{p}=\mu_{y}+\sigma_{y} k_{p}^{*}$, and we require $x_{p}<y_{p}$ to hold for all $p \in[0,1]$. Then when $p \downarrow 0 \Rightarrow k_{0}^{*} \rightarrow \infty$ and $p \uparrow 1 \Rightarrow k_{1}^{*} \rightarrow-\infty$. If $S\left(k_{p}^{*}\right)=y_{p}-x_{p}$, then

$$
S\left(k_{p}^{*}\right)=\mu_{y}+\sigma_{y} k_{p}^{*}-\mu_{x}-\sigma_{x} k_{p}^{*} .
$$

For $k_{p}^{*}=M$ with $M \uparrow \infty$,

$$
\begin{aligned}
S(M) & =\mu_{y}-\mu_{x}+\sigma_{y} M-\sigma_{x} M \\
& \approx M\left(\sigma_{y}-\sigma_{x}\right) \text { as } \quad M \rightarrow \infty,
\end{aligned}
$$

we need $S(M)>0$ as $M \uparrow \infty$ then we get $\sigma_{y}-\sigma_{x}>0$. For $k_{p}^{*}=-M$ with $M \uparrow \infty$,

$$
\begin{aligned}
S(-M) & =\mu_{y}-\mu_{x}-\sigma_{y} M+\sigma_{x} M \\
& \approx M\left(\sigma_{x}-\sigma_{y}\right) \text { as } M \rightarrow \infty,
\end{aligned}
$$

we need $S(-M)>0$ as $M \uparrow \infty$ then we get $\sigma_{x}-\sigma_{y}>0$.

As $S(-M)>0 \Rightarrow \sigma_{x}>\sigma_{y}$ and $S(M)>0 \Rightarrow \sigma_{x}<\sigma_{y}$, the only possible solution is $\sigma_{x}=\sigma_{y}$. Then $S\left(k_{p}^{*}\right) \geq 0$ holds for all $p$ when $\sigma_{x}=\sigma_{y}$ and $\mu_{x}<\mu_{y}$ as $S($.$) then does not$ depend on $p$. 
Proof Case 2: $\xi_{x}=\xi_{y}=\xi>0$.

Let $k_{p}=\frac{1}{\xi}\left[1-(-\log p)^{-\xi}\right]$ then $x_{p}=\mu_{x}+\sigma_{x} k_{p}$ and $y_{p}=\mu_{y}+\sigma_{y} k_{p}$, and we require $x_{p}<y_{p}$ to hold for all $p \in[0,1]$. Then when $p=0 \Rightarrow k_{0}=\frac{1}{\xi}>0$ and $p \rightarrow 1 \Rightarrow k_{1} \rightarrow-\infty$. If $S\left(k_{p}\right)=y_{p}-x_{p}$, then

$$
S\left(k_{p}\right)=\mu_{y}+\sigma_{y} k_{p}-\mu_{x}-\sigma_{x} k_{p} .
$$

For $k_{p}=-M$ with $M \uparrow \infty$,

$$
\begin{aligned}
S(-M) & =\mu_{y}-\mu_{x}-\sigma_{y} M+\sigma_{x} M \\
& \sim M\left(\sigma_{x}-\sigma_{y}\right) \text { as } M \rightarrow \infty
\end{aligned}
$$

we need $S(-M)>0$ as $M \uparrow \infty$ then we get $\sigma_{x}-\sigma_{y}>0$. For $k_{0}=\frac{1}{\xi}$ then

$$
S\left(\frac{1}{\xi}\right)=\mu_{y}-\mu_{x}+\frac{\sigma_{y}}{\xi}-\frac{\sigma_{x}}{\xi}
$$

we need $S\left(\frac{1}{\xi}\right)>0$, then we get

$$
\mu_{y}-\mu_{x}+\frac{\sigma_{y}-\sigma_{x}}{\xi}>0 .
$$

We do not need to check for $0<p<1$ because $S\left(k_{p}\right)$ is linear in $k_{p}$ so its extreme value over a range of $k_{p}$ occur at the endpoints of $k_{p}$.

Proof Case 3: $\xi_{x}=\xi_{y}=\xi<0$.

Let $k_{p}=\frac{1}{\xi}\left[1-(-\log p)^{-\xi}\right]$ then $x_{p}=\mu_{x}+\sigma_{x} k_{p}$ and $y_{p}=\mu_{y}+\sigma_{y} k_{p}$, and we require $x_{p}<y_{p}$ to hold for all $p \in[0,1]$. Then when $p \rightarrow 0 \Rightarrow k_{0} \rightarrow \infty$ and $p=1 \Rightarrow k_{1}=\frac{1}{\xi}$. If $S\left(k_{p}\right)=y_{p}-x_{p}$, then using Equation (5), for $k_{p}=M$ with $M \uparrow \infty$,

$$
\begin{aligned}
S(M) & =\mu_{y}-\mu_{x}+\sigma_{y} M-\sigma_{x} M \\
& \sim M\left(\sigma_{y}-\sigma_{x}\right) \text { as } \quad M \rightarrow \infty,
\end{aligned}
$$

we need $S(M)>0$ as $M \uparrow \infty$ then we get $\sigma_{x}<\sigma_{y}$. For $k_{1}=\frac{1}{\xi}$ then

$$
S\left(\frac{1}{\xi}\right)=\mu_{y}-\mu_{x}+\frac{\sigma_{y}}{\xi}-\frac{\sigma_{x}}{\xi}
$$

we need $S\left(\frac{1}{\xi}\right)>0$, then we get

$$
\mu_{y}-\mu_{x}+\frac{\sigma_{y}-\sigma_{x}}{\xi}>0 .
$$

As $S\left(k_{p}\right)=0$, is linear in $k_{p}$ so its extreme value over a range of $k_{p}$ occur at the endpoints of $k_{p}$. 
Proof Case $4: \xi_{x}=0, \xi_{y}>0$.

Let $k_{x ; p}=\log (-\log p)$ and $k_{y ; p}=\frac{1}{\xi_{y}}\left[1-(-\log p)^{-\xi_{y}}\right]$ then $x_{p}=\mu_{x}+\sigma_{x} k_{x ; p}$ and $y_{p}=$ $\mu_{y}+\sigma_{y} k_{y ; p}$, and we require $x_{p}<y_{p}$ to hold for all $p \in[0,1]$. Then when $p \downarrow 0 \Rightarrow k_{x ; 0} \rightarrow \infty$ and $p=0 \Rightarrow k_{y ; 0}=\frac{1}{\xi_{y}}>0$; and when $p \uparrow 1 \Rightarrow k_{x ; 1} \rightarrow-\infty$ and $k_{y ; 1} \rightarrow-\infty$.

If $S\left(k_{x ; p}\right)=y\left(k_{x ; p}\right)-x\left(k_{x ; p}\right)$, using the transformation $-\log p=\exp \left(k_{x ; p}\right)$, then

$$
S\left(k_{x ; p}\right)=\mu_{y}+\frac{\sigma_{y}}{\xi_{y}}\left[1-\exp \left(-k_{x ; p} \xi_{y}\right)\right]-\mu_{x}-\sigma_{x} k_{x ; p} .
$$

For $k_{x ; p}=N$ with $N \uparrow \infty$,

$$
\begin{aligned}
S(N) & =\mu_{y}+\frac{\sigma_{y}}{\xi_{y}}\left[1-\exp \left(-N \xi_{y}\right)\right]-\mu_{x}-\sigma_{x} N \\
& \approx \frac{\sigma_{y}}{\xi_{y}}\left[1-\exp \left(-N \xi_{y}\right)\right]-\sigma_{x} N \\
& \approx-\frac{\sigma_{y}}{\xi_{y}} \exp \left(-N \xi_{y}\right)-\sigma_{x} N \quad \downarrow-\infty,
\end{aligned}
$$

as $\xi_{y}>0$ and $\frac{\exp \left(-N \xi_{y}\right)}{N} \rightarrow 0$. For $k_{x ; p}=-N$ with $N \uparrow \infty$,

$$
\begin{aligned}
S(-N) & =\mu_{y}+\frac{\sigma_{y}}{\xi_{y}}\left[1-\exp \left(+N \xi_{y}\right)\right]-\mu_{x}+\sigma_{x} N \\
& \approx \frac{\sigma_{y}}{\xi_{y}}\left[1-\exp \left(+N \xi_{y}\right)\right]+\sigma_{x} N \\
& \approx-\frac{\sigma_{y}}{\xi_{y}} \exp \left(+N \xi_{y}\right)+\sigma_{x} N \quad \downarrow-\infty,
\end{aligned}
$$

as $\xi_{y}>0$ and $\frac{\exp \left(N \xi_{y}\right)}{N} \rightarrow \infty$. These conditions $\left(\xi_{x}=0\right.$ and $\left.\xi_{y}>0\right)$ are impossible to give us $S\left(k_{x ; p}\right)>0$ for all range of $k_{x ; p} \in(-\infty, \infty)$.

Proof Case 5: $\xi_{x}=0, \xi_{y}<0$.

Let $k_{x ; p}=\log (-\log p)$ and $k_{y ; p}=\frac{1}{\xi_{y}}\left[1-(-\log p)^{-\xi_{y}}\right]$ then $x_{p}=\mu_{x}+\sigma_{x} k_{x ; p}$ and $y_{p}=\mu_{y}+\sigma_{y} k_{y ; p}$, and we require $x_{p}<y_{p}$ to hold for all $p \in[0,1]$.

Then when $p \downarrow 0 \Rightarrow k_{x ; 0} \rightarrow \infty$ and $k_{y ; 0} \rightarrow \infty$, and $p \uparrow 1 \Rightarrow k_{x ; 1}=-\infty$ and when $p=1 \Rightarrow k_{y ; 1}=\frac{1}{\xi_{y}}$. If $S\left(k_{x ; p}\right)=y_{p}-x_{p}$, then we get Equation (6). For $k_{x ; p}=N$ with $N \uparrow \infty$, we get

$$
\begin{aligned}
S(N) & =\mu_{y}+\frac{\sigma_{y}}{\xi_{y}}\left[1-\exp \left(-N \xi_{y}\right)\right]-\mu_{x}-\sigma_{x} N \\
& \approx \frac{\sigma_{y}}{\xi_{y}}\left[1-\exp \left(-N \xi_{y}\right)\right]-\sigma_{x} N \\
& \approx-\frac{\sigma_{y}}{\xi_{y}} \exp \left(-N \xi_{y}\right)-\sigma_{x} N \quad \uparrow \infty,
\end{aligned}
$$


as $\frac{\exp \left(-N \xi_{y}\right)}{N} \rightarrow \infty$. For $k_{x ; p}=-N$ with $N \uparrow \infty$,

$$
\begin{aligned}
S(-N) & =\mu_{y}+\frac{\sigma_{y}}{\xi_{y}}\left[1-\exp \left(+N \xi_{y}\right)\right]-\mu_{x}+\sigma_{x} N \\
& \approx \frac{\sigma_{y}}{\xi_{y}}\left[1-\exp \left(+N \xi_{y}\right)\right]+\sigma_{x} N \\
& \approx \sigma_{x} N \quad \uparrow \infty .
\end{aligned}
$$

To make sure $S>0$ for all range of $k_{x ; p}$, we check whether the $S$ has minimum value in the range $k_{x ; p} \in(-\infty, \infty)$,

$$
S^{\prime}\left(k_{x ; p}\right)=\sigma_{y} \exp \left(-k_{x} \xi_{y}\right)-\sigma_{x}
$$

When, $S^{\prime}\left(k^{*}\right)=\sigma_{y} \exp \left(-k^{*} \xi_{y}\right)-\sigma_{x}=0$ then $k^{*}=-\frac{1}{\xi_{y}} \log \left(\frac{\sigma_{x}}{\sigma_{y}}\right)$, put inside $S^{\prime \prime}($.$) we get$ $S^{\prime \prime}\left(k^{*}\right)=-\sigma_{y} \xi_{y}\left(\frac{\sigma_{x}}{\sigma_{y}}\right)>0$. The $S($.$) function is having a minimum value.$

$$
\begin{aligned}
S\left(k^{*}\right) & =\mu_{y}+\frac{\sigma_{y}}{\xi_{y}}\left\{1-\exp \left[\log \left(\frac{\sigma_{x}}{\sigma_{y}}\right)\right]\right\}-\mu_{x}+\frac{\sigma_{x}}{\xi_{y}} \log \left(\frac{\sigma_{x}}{\sigma_{y}}\right) \\
& =\mu_{y}+\frac{\sigma_{y}}{\xi_{y}}\left(1-\frac{\sigma_{x}}{\sigma_{y}}\right)-\mu_{x}+\frac{\sigma_{x}}{\xi_{y}} \log \left(\frac{\sigma_{x}}{\sigma_{y}}\right) .
\end{aligned}
$$

In order to satisfy $S()>$.0 then $\mu_{y}+\frac{\sigma_{y}}{\xi_{y}}\left(1-\frac{\sigma_{x}}{\sigma_{y}}\right)-\mu_{x}+\frac{\sigma_{x}}{\xi_{y}} \log \left(\frac{\sigma_{x}}{\sigma_{y}}\right)>0$.

Proof Case 6: $0<\xi_{x}, \quad \xi_{y}=0$.

Let $k_{y ; p}=\log (-\log p)$ and $k_{x ; p}=\frac{1}{\xi_{x}}\left[1-(-\log p)^{-\xi_{x}}\right]$ then $y_{p}=\mu_{y}+\sigma_{y} k_{y ; p}$ and $x_{p}=\mu_{x}+\sigma_{x} k_{x ; p}$, and we require $x_{p}<y_{p}$ to hold for all $p \in[0,1]$. Using a transformation of $-\log p=\exp \left(k_{y ; p}\right)$, we get $x_{p}=\mu_{x}+\frac{\sigma_{x}}{\xi_{x}}\left\{1-\left[\exp \left(k_{y ; p}\right)\right]^{-\xi) x}\right\}$. Then when $p \downarrow 0 \Rightarrow k_{y ; 0} \rightarrow \infty$ and $p \uparrow 1 \Rightarrow k_{y ; 1} \rightarrow-\infty$. If $S\left(k_{y ; p}\right)=y_{p}-x_{p}$, then

$$
S\left(k_{y ; p}\right)=\mu_{y}+\sigma_{y} k_{y ; p}-\mu_{x}+\frac{\sigma_{x}}{\xi_{x}}\left[1-\exp \left(-\xi_{x} k_{y ; p}\right)\right] .
$$

For $k_{y ; p}=M, M \uparrow \infty$,

$$
\begin{aligned}
S(M) & =\mu_{y}+\sigma_{y} M-\mu_{x}+\frac{\sigma_{x}}{\xi_{x}}\left[1-\exp \left(-\xi_{x} M\right)\right] \\
& \approx \sigma_{y} M-\frac{\sigma_{x}}{\xi_{x}} \exp \left(-\xi_{x} M\right) \quad \uparrow \infty,
\end{aligned}
$$

as $\exp \left(-\xi_{x} M\right) \rightarrow 0$. For $k_{y ; p}=-M, M \uparrow \infty$,

$$
\begin{aligned}
S(-M) & =\mu_{y}-\sigma_{y} M-\mu_{x}+\frac{\sigma_{x}}{\xi_{x}}\left[1-\exp \left(\xi_{x} M\right)\right] \\
& \approx-\sigma_{y} M-\frac{\sigma_{x}}{\xi_{x}} \exp \left(\xi_{x} M\right) \quad \downarrow-\infty,
\end{aligned}
$$


as $\exp \left(\xi_{x} M\right) \rightarrow \infty$. The condition $S\left(k_{y ; p}\right)>0$ is not covered for all range $k_{y ; p} \in(-\infty, \infty)$.

Proof Case 7: $\xi_{x}<0, \quad \xi_{y}=0$.

Let $k_{y ; p}=\log (-\log p)$ and $k_{x ; p}=\frac{1}{\xi_{x}}\left[1-(-\log p)^{-\xi_{x}}\right]$ then $y_{p}=\mu_{y}+\sigma_{y} k_{y ; p}$ and $x_{p}=\mu_{x}+\sigma_{x} k_{x ; p}$, and we require $x_{p}<y_{p}$ to hold for all $p \in[0,1]$. Using a transformation of $-\log p=\exp \left(k_{y ; p}\right)$, we get $x_{p}=\mu_{x}+\frac{\sigma_{x}}{\xi_{x}}\left\{1-\left[\exp \left(k_{y ; p}\right)\right]^{-\xi_{x}}\right\}$. Then when $p \downarrow 0 \Rightarrow k_{y ; 0} \rightarrow \infty$ and $p \uparrow 1 \Rightarrow k_{y ; 1} \rightarrow-\infty$. If $S\left(k_{y ; p}\right)=y_{p}-x_{p}$, then it similar to Equation (7). For $k_{y ; p}=N$ with $N \uparrow \infty$,

$$
\begin{aligned}
S(N) & =\mu_{y}+\sigma_{y} N-\mu_{x}-\frac{\sigma_{x}}{\xi_{x}}\left[1-\exp \left(-N \xi_{x}\right)\right] \\
& \approx \sigma_{y} N-\frac{\sigma_{x}}{\xi_{x}}\left[1-\exp \left(-N \xi_{x}\right)\right] \\
& \approx \sigma_{y} N+\frac{\sigma_{x}}{\xi_{x}} \exp \left(-N \xi_{x}\right) \quad \uparrow \infty
\end{aligned}
$$

as $0>\xi_{x}$ and $\frac{\exp \left(-N \xi_{y}\right)}{N} \rightarrow \infty$. For $k_{y ; p}=-N$ with $N \uparrow \infty$,

$$
\begin{aligned}
S(-N) & =\mu_{y}-\sigma_{y} N-\mu_{x}-\frac{\sigma_{x}}{\xi_{x}}\left[1-\exp \left(+N \xi_{x}\right)\right] \\
& \approx-\sigma_{y} N+\frac{\sigma_{x}}{\xi_{x}} \exp \left(+N \xi_{x}\right) \quad \downarrow-\infty
\end{aligned}
$$

as $\xi_{y}<0$ and $\frac{\exp \left(N \xi_{y}\right)}{N} \rightarrow 0$.

The condition of $S\left(k_{y ; p}\right)>0$ is not satisfied for all range of $k_{y ; p} \in(-\infty, \infty)$.

Proof Case 8: $0<\xi_{x}<\xi_{y}$.

Let $k_{x ; p}=\frac{1}{\xi_{x}}\left[1-(-\log p)^{-\xi_{x}}\right]$ then $y_{p}=\mu_{y}+\frac{\sigma_{y}}{\xi_{y}}\left[1-\left(1-k_{x ; p} \xi_{x}\right)^{\xi_{y} / \xi_{x}}\right]$, and we require $x_{p}<y_{p}$ to hold for all $p \in[0,1]$. Then when $p=0 \Rightarrow k_{x ; 0}=\frac{1}{\xi_{x}}>0$ and $p \uparrow 1 \Rightarrow k_{x ; 1} \rightarrow$ $-\infty$. If $S\left(k_{x ; p}\right)=y_{p}-x_{p}$, then

$$
S\left(k_{x ; p}\right)=\mu_{y}+\frac{\sigma_{y}}{\xi_{y}}\left[1-\left(1-k_{x ; p} \xi_{x}\right)^{\xi_{y} / \xi_{x}}\right]-\mu_{x}-\sigma_{x} k_{x ; p} .
$$

For $k_{x ; p}=-M$ with $M \uparrow \infty$

$$
\begin{aligned}
S(-M) & \approx \mu_{y}+\frac{\sigma_{y}}{\xi_{y}}\left[1-\left(M \xi_{x}\right)^{\xi_{y} / \xi_{x}}\right]-\mu_{x}+\sigma_{x} M \\
& \approx \frac{\sigma_{y}}{\xi_{y}}\left[1-\left(M \xi_{x}\right)^{\xi_{y} / \xi_{x}}\right]+\sigma_{x} M \\
& \sim-\frac{\sigma_{y}}{\xi_{y}} \xi_{x}^{\xi_{y} / \xi_{x}} M^{\xi_{y} / \xi_{x}}+\sigma_{x} M \downarrow-\infty \quad \text { as } \quad \frac{\xi_{y}}{\xi_{x}}>1 \quad \text { so } \quad \frac{M^{\xi_{y} / \xi_{x}}}{M} \rightarrow \infty .
\end{aligned}
$$

The result for $S(-M)>0$ is not satisfied unless $\xi_{y}=\xi_{x}$ which is covered in Cases 1-3. 
Proof Case 9: $0<\xi_{y}<\xi_{x}$.

Let

$$
k_{y}=\frac{1}{\xi_{y}}\left[1-(-\log p)^{-\xi_{y}}\right] \text { and } k_{x}=\frac{1}{\xi_{x}}\left[1-(-\log p)^{-\xi_{x}}\right]
$$

then $y_{p}=\mu_{y}+\sigma_{y} k_{y ; p}$ and $x_{p}=\mu_{x}+\sigma_{x} k_{x ; p}$, and we require $x_{p}<y_{p}$ to hold for all $p \in[0,1]$. Then when $p=0 \Rightarrow k_{x ; 0}=\frac{1}{\xi_{x}}>0$ and $p \uparrow 1 \Rightarrow k_{x ; 1} \rightarrow-\infty$. If $S\left(k_{y ; p}\right)=y_{p}-x_{p}$, then get Equation (8). For $k_{x ; p}=-M$ with $M \uparrow \infty$,

$$
\begin{aligned}
S(-M) & =\mu_{y}+\frac{\sigma_{y}}{\xi_{y}}\left[1-\left(1+M \xi_{x}\right)^{\xi_{y} / \xi_{x}}\right]-\mu_{x}+\sigma_{x} M \\
& \approx-\frac{\sigma_{y}}{\xi_{y}}\left(1+M \xi_{x}\right)^{\xi_{y} / \xi_{x}}+\sigma_{x} M \quad \uparrow \infty \quad \frac{\xi_{y}}{\xi_{x}}<1 ; \frac{M^{\xi_{y} / \xi_{x}}}{M} \rightarrow 0 .
\end{aligned}
$$

For $k_{x ; p}=\frac{1}{\xi_{x}}$

$$
\begin{aligned}
S\left(\frac{1}{\xi_{x}}\right) & =\mu_{y}+\frac{\sigma_{y}}{\xi_{y}}\left[1-\left(1-\frac{1}{\xi_{x}} \xi_{x}\right)^{\xi) y / \xi_{x}}\right]-\mu_{x}+\sigma_{x} \frac{1}{\xi_{x}} \\
& =\mu_{y}+\frac{\sigma_{y}}{\xi_{y}}-\mu_{x}-\frac{\sigma_{x}}{\xi_{x}}>0 .
\end{aligned}
$$

We need to check whether the $S\left(k_{x ; p}\right)$ has a minimum value in the range $k_{x ; p} \in\left(-\infty, \frac{1}{\xi_{x}}\right)$. We get

$$
S^{\prime}\left(k_{x ; p}\right)=\sigma_{y}\left(1-k_{x ; p} \xi_{x}\right)^{\frac{\xi_{y}}{\xi_{x}}-1}-\sigma_{x}
$$

suppose $S^{\prime}\left(k_{x ; p}^{*}\right)=0$, we get $k_{x}^{*}=\frac{1}{\xi_{x}}\left[1-\left(\frac{\sigma_{x}}{\sigma_{y}}\right)^{\xi_{x} /\left(\xi_{y}-\xi_{x}\right)}\right]$.

$$
\begin{aligned}
S\left(k_{x ; p}^{*}\right) & =\mu_{y}+\frac{\sigma_{y}}{\xi_{y}}\left[1-\left(\frac{\sigma_{x}}{\sigma_{y}}\right)^{\xi_{y} /\left(\xi_{y}-\xi_{x}\right)}\right]-\mu_{x}-\frac{\sigma_{x}}{\xi_{x}}\left[1-\left(\frac{\sigma_{x}}{\sigma_{y}}\right)^{\xi_{x} /\left(\xi_{y}-\xi_{x}\right)}\right] \\
& =\mu_{y}+\frac{\sigma_{y}}{\xi_{y}}-\mu_{x}-\frac{\sigma_{x}}{\xi_{x}}+\sigma_{x}^{\xi_{y} /\left(\xi_{y}-\xi_{x}\right)} \sigma_{y}^{-\xi_{x} /\left(\xi_{y}-\xi_{x}\right)}\left(\frac{1}{\xi_{x}}-\frac{1}{\xi_{y}}\right) \\
& =\mu_{y}+\frac{\sigma_{y}}{\xi_{y}}-\mu_{x}-\frac{\sigma_{x}}{\xi_{x}}+c\left(\frac{1}{\xi_{x}}-\frac{1}{\xi_{y}}\right),
\end{aligned}
$$

where $c=\sigma_{x}^{\xi_{y} /\left(\xi_{y}-\xi_{x}\right)} \sigma_{y}^{-\xi_{x} /\left(\xi_{y}-\xi_{x}\right)}>0$ and $\frac{1}{\xi_{x}}-\frac{1}{\xi_{y}}<0$.

$$
\begin{aligned}
S^{\prime \prime}\left(k_{x ; p}^{*}\right) & =-\sigma_{y}\left(\xi_{y}-\xi_{x}\right)\left(1-k_{x ; p}^{*} \xi_{x}\right)\left(\xi_{y} / \xi_{x}\right)-2 \\
& =-\sigma_{y}\left(\xi_{y}-\xi_{x}\right)\left(\frac{\sigma_{x}}{\sigma_{y}}\right)^{\frac{\xi_{y}-2 \xi_{x}}{\xi_{y}-\xi_{x}}}>0 \quad \text { as } \quad \xi_{y}<\xi_{x} .
\end{aligned}
$$

Although $S($.$) has a minimum value for all range of k_{x ; p}$ but the condition of $S\left(k_{x ; p}\right)>0$ is not fully satisfied, unless $\xi_{x}=\xi_{y}$ which is covered in Cases 1-3. 
Proof Case 10: $\xi_{y}<\xi_{x}<0$.

Let $k_{y ; p}=\frac{1}{\xi_{y}}\left[1-(-\log p)^{-\xi_{y}}\right]$ and $k_{x ; p}=\frac{1}{\xi_{x}}\left[1-(-\log p)^{-\xi_{x}}\right]$ then $y_{p}=\mu_{y}+\sigma_{y} k_{y ; p}$ and $x_{p}=\mu_{x}+\sigma_{x} k_{x ; p}$, and we require $x_{p}<y_{p}$ to hold for all $p \in[0,1]$. Then when $p \downarrow 0 \Rightarrow k_{y ; 0} \rightarrow \infty$ and $k_{x ; 0} \rightarrow \infty$. When $p=1 \Rightarrow k_{y ; 1}=\frac{1}{\xi_{y}}$ and $k_{x ; 1}=\frac{1}{\xi_{x}}$. If $S\left(k_{x ; p}\right)=y_{p}-x_{p}$, then $S\left(k_{x ; p}\right)$ is similar to Equation (8). For $k_{x ; p}=M$, with $M \uparrow \infty$,

$$
S(M) \approx-\frac{\sigma_{y}}{\xi_{y}}\left(1-M \xi_{x}\right)^{\xi_{y} / \xi_{x}}-\sigma_{x} M \quad \uparrow \infty .
$$

For $k_{x ; p}=\frac{1}{\xi_{x}}$,

$$
S\left(\frac{1}{\xi_{x}}\right)=\mu_{y}+\frac{\sigma_{y}}{\xi_{y}}-\mu_{x}-\frac{\sigma_{x}}{\xi_{x}}
$$

where $S\left(k_{x ; p}\right)>0$ only if $\mu_{y}+\frac{\sigma_{y}}{\xi_{y}}-\mu_{x}-\frac{\sigma_{x}}{\xi_{x}}>0$.

We need to check whether the $S\left(k_{x ; p}\right)$ has a positive minimum value in the range of $k_{x ; p}$. From Equation (9) calculating for the critical point, for the minimum value of the $S\left(k_{x ; p}\right)$, we found that $c\left(\frac{1}{\xi_{x}}-\frac{1}{\xi_{y}}\right)<0$ where $c=\sigma_{x}^{\xi_{y} /\left(\xi_{y}-\xi_{x}\right)} \sigma_{y}^{-\xi_{x} /\left(\xi_{y}-\xi_{x}\right)}>0$. The condition of $S\left(k_{x ; p}\right)>0$ is not fully satisfied unless $\xi_{x}=\xi_{y}$ which is covered in Cases 1-3.

Proof Case 11: $\xi_{x}<\xi_{y}<0$.

Let $k_{y ; p}=\frac{1}{\xi_{y}}\left[1-(-\log p)^{-\xi_{y}}\right]$ and $k_{x ; p}=\frac{1}{\xi_{x}}\left[1-(-\log p)^{-\xi_{x}}\right]$ then $y_{p}=\mu_{y}+\sigma_{y} k_{y ; p}$ and $x_{p}=\mu_{x}+\sigma_{x} k_{x ; p}$, and we require $x_{p}<y_{p}$ to hold for all $p \in[0,1]$. Then when $p \downarrow 0 \Rightarrow k_{y ; 0} \rightarrow \infty$ and $k_{x ; 0} \rightarrow \infty$. When $p=1 \Rightarrow k_{y ; 1}=\frac{1}{\xi_{y}}$ and $k_{x ; 1}=\frac{1}{\xi_{x}}$. If $S\left(k_{x ; p}\right)=y_{p}-x_{p}$, then $S\left(k_{x ; p}\right)$ is similar to Equation (8). For $k_{x ; p}=M, M \uparrow \infty$

$$
\begin{aligned}
S(M) & =\mu_{y}+\frac{\sigma_{y}}{\xi_{y}}\left[1-\left(1-M \xi_{x}\right)^{\xi_{y} / \xi_{x}}\right]-\mu_{x}-\sigma_{x} M \\
& \approx-\frac{\sigma_{y}}{\xi_{y}}\left(1-M \xi_{x}\right)^{\xi_{y} / \xi_{x}}-\sigma_{x} M \quad \downarrow-\infty .
\end{aligned}
$$

For $k_{x ; p}=\frac{1}{\xi_{x}}$

$$
\begin{aligned}
S\left(\frac{1}{\xi_{x}}\right) & =\mu_{y}+\frac{\sigma_{y}}{\xi_{y}}\left[1-\left(1-\frac{1}{\xi_{x}} \xi_{x}\right)^{\xi_{y} / \xi_{x}}\right]-\mu_{x}-\sigma_{x} \frac{1}{\xi_{x}} \\
& =\mu_{y}+\frac{\sigma_{y}}{\xi_{y}}-\mu_{x}-\frac{\sigma_{x}}{\xi_{x}} .
\end{aligned}
$$

The condition for $S\left(k_{x ; p}\right)>0$ is not satisfied for $k_{x ; p} \rightarrow \infty$. 
Proof Case 12: $\xi_{y}<0<\xi_{x}$.

Let $k_{x ; p}=\frac{1}{\xi_{x}}\left[1-(-\log p)^{-\xi_{x}}\right]$ and $k_{y ; p}=\frac{1}{\xi_{y}}\left[1-(-\log p)^{-\xi_{y}}\right]$ then $x_{p}=\mu_{x}+\sigma_{x} k_{x ; p}$ and $y_{p}=\mu_{y}+\sigma_{y} k_{y ; p}$, and we require $x_{p}<y_{p}$ to hold for all $p \in[0,1]$. When $p=0 \Rightarrow k_{x ; 0}=$ $\frac{1}{\xi_{x}}>0$ and $p \downarrow 0 \Rightarrow k_{y ; 0} \rightarrow \infty$, and when $p \uparrow 1 \Rightarrow k_{x ; 1} \rightarrow-\infty$ and $p=1 \Rightarrow k_{y ; 1}=\frac{1}{\xi_{y}}<0$. If $S\left(k_{x ; p}\right)=y_{p}-x_{p}$, then we get Equation (8). For $k_{x ; p}=-M, M \uparrow \infty$, we get

$$
\begin{aligned}
S(-M) & =\mu_{y}+\frac{\sigma_{y}}{\xi_{y}}\left[1-\left(1+M \xi_{x}\right)^{\xi_{y} / \xi_{x}}\right]-\mu_{x}+\sigma_{x} M \\
& \approx-\frac{\sigma_{y}}{\xi_{y}}\left(1+M \xi_{x}\right)^{\xi_{y} / \xi_{x}}+\sigma_{x} M \\
& \approx-\frac{\sigma_{y}}{\xi_{y}} \xi_{x}^{\xi_{y} / \xi_{x}} M^{\xi_{y} / \xi_{x}}+\sigma_{x} M \quad \uparrow \infty .
\end{aligned}
$$

For $k_{x ; p}=\frac{1}{\xi_{x}}-\xi$ as $\xi \downarrow 0$, we get

$$
\begin{aligned}
S\left(\frac{1}{\xi_{x}}-\xi\right) & =\mu_{y}+\frac{\sigma_{y}}{\xi_{y}}\left\{1-\left[1-\left(\frac{1}{\xi_{x}}-\xi\right) \xi_{x}\right]^{\xi_{y} / \xi_{x}}\right\}-\mu_{x}-\sigma_{x}\left(\frac{1}{\xi_{x}}-\xi\right) \\
& =\mu_{y}+\frac{\sigma_{y}}{\xi_{y}}\left[1-\left(\xi \xi_{x}\right)^{\xi_{y} / \xi_{x}}\right]-\mu_{x}-\frac{\sigma_{x}}{\xi_{x}}+\xi \sigma_{x} \\
& \approx-\frac{\sigma_{y}}{\xi_{y}} \xi^{\xi_{y} / \xi_{x}} \uparrow \infty \text { as } \xi \downarrow 0, \frac{\xi_{y}}{\xi_{x}}<0 .
\end{aligned}
$$

We need to check whether the $S($.$) has a minimum value in the range k_{x ; p} \in\left(-\infty, \frac{1}{\xi_{x}}\right)$. We get

$$
S^{\prime}\left(k_{x ; p}\right)=\sigma_{y}\left(1-k_{x ; p} \xi_{x}\right)^{\left(\xi_{y}-\xi_{x}\right) / \xi_{x}}-\sigma_{x}
$$

Let $S^{\prime}\left(k_{x ; p}^{*}\right)=0$ we get $k_{x ; p}^{*}=\frac{1}{\xi_{x}}\left[1-\left(\frac{\sigma_{x}}{\sigma_{y}}\right)^{\xi_{x} /\left(\xi_{y}-\xi_{x}\right)}\right]$. Then, we need to prove that $k_{x ; p}^{*} \in\left(-\infty, \frac{1}{\xi_{x}}\right)$

$$
\begin{aligned}
k_{x ; p}^{*} & \leq \frac{1}{\xi_{x}} \\
\frac{1}{\xi_{x}}\left[1-\left(\frac{\sigma_{x}}{\sigma_{y}}\right)^{\xi_{x} /\left(\xi_{y}-\xi_{x}\right)}\right] & \leq \frac{1}{\xi_{x}} \\
{\left[1-\left(\frac{\sigma_{x}}{\sigma_{y}}\right)^{\xi_{x} /\left(\xi_{y}-\xi_{x}\right)}\right] } & \leq 1 \\
0 & \leq\left(\frac{\sigma_{x}}{\sigma_{y}}\right)^{\xi_{x} /\left(\xi_{y}-\xi_{x}\right)} .
\end{aligned}
$$

We find that $k^{*} \in\left(-\infty, \frac{1}{\xi_{x}}\right)$ for $0<p<1$. When

$$
S^{\prime \prime}\left(k_{x ; p}\right)=\sigma_{y}\left(\xi_{y}-\xi_{x}\right)\left(1-k_{x ; p} \xi_{x}\right)^{\left(\xi_{y} / \xi_{x}\right)-2},
$$


put the $k_{x ; p}^{*}$ we get

$$
S^{\prime \prime}\left(k_{x ; p}^{*}\right)=-\sigma_{y}\left(\xi_{y}-\xi_{x}\right)\left(\frac{\sigma_{x}}{\sigma_{y}}\right)^{\left(\xi_{y}-2 \xi_{x}\right) /\left(\xi_{y}-\xi_{x}\right)}>0 \quad \text { as } \quad \xi_{y}<\xi_{x}
$$

which is a minima point exist in the range of $k_{x ; p}$ as $S^{\prime \prime}\left(k_{x ; p}^{*}\right)>0$. We check whether the $S\left(k_{x ; p}^{*}\right)$ has a positive value,

$$
\begin{aligned}
S\left(k_{x}^{*}\right)= & \mu_{y}+\frac{\sigma_{y}}{\xi_{y}}\left[1-\left(\frac{\sigma_{x}}{\sigma_{y}}\right)^{\xi_{y} /\left(\xi_{y}-\xi_{x}\right)}\right] \\
& -\mu_{x}-\frac{\sigma_{x}}{\xi_{x}}\left[1-\left(\frac{\sigma_{x}}{\sigma_{y}}\right)^{\xi_{x} /\left(\xi_{y}-\xi_{x}\right)}\right] \\
= & \mu_{y}+\frac{\sigma_{y}}{\xi_{y}}-\mu_{x} \\
& -\frac{\sigma_{x}}{\xi_{x}}+\sigma_{x}^{\xi_{y} /\left(\xi_{y}-\xi_{x}\right)} \sigma_{y}^{-\xi_{x} /\left(\xi_{y}-\xi_{x}\right)}\left(\frac{1}{\xi_{x}}-\frac{1}{\xi_{y}}\right) \\
= & \mu_{y}+\frac{\sigma_{y}}{\xi_{y}}-\mu_{x}-\frac{\sigma_{x}}{\xi_{x}}+c\left(\frac{1}{\xi_{x}}-\frac{1}{\xi_{y}}\right),
\end{aligned}
$$

where $c=\sigma_{x}^{\xi_{y} /\left(\xi_{y}-\xi_{x}\right)} \sigma_{y}^{-\xi_{x} /\left(\xi_{y}-\xi_{x}\right)}>0$. If $\mu_{y}+\frac{\sigma_{y}}{\sigma_{y}}-\mu_{x}-\frac{\sigma_{x}}{\xi_{x}}>c\left(\frac{1}{\xi_{x}}-\frac{1}{\xi_{y}}\right)$ then $S\left(k_{x}^{*}\right)>0$.

Proof Case 13: $\xi_{x}<0<\xi_{y}$.

Let $k_{x ; p}=\frac{1}{\xi_{x}}\left[1-(-\log p)^{-\xi_{x}}\right]$ and $k_{y ; p}=\frac{1}{\xi_{y}}\left[1-(-\log p)^{-\xi_{y}}\right]$ then $x_{p}=\mu_{x}+\sigma_{x} k_{x ; p}$ and $y_{p}=\mu_{y}+\sigma_{y} k_{y ; p}$, and we require $x_{p}<y_{p}$ to hold for all $p \in[0,1]$. When $p \downarrow 0 \Rightarrow k_{x ; 0} \rightarrow \infty$ and $p=0 \Rightarrow k_{y ; 0}=\frac{1}{\xi_{y}}>0$, and when $p=1 \Rightarrow k_{x ; 1}=\frac{1}{\xi_{x}}<0$ and $p \uparrow 1 \Rightarrow k_{y ; 1} \rightarrow-\infty$. If $S\left(\hat{k}_{p}\right)=y_{p}-x_{p}$, then we get Equation (8). For $k_{x ; p}=M$ with $M \uparrow \infty$,

$$
\begin{aligned}
S(M) & =\mu_{y}+\frac{\sigma_{y}}{\xi_{y}}\left[1-\left(1-M \xi_{x}\right)^{\xi_{y} / \xi_{x}}\right]-\mu_{x}-\sigma_{x} M \\
& \approx-\frac{\sigma_{y}}{\xi_{y}}\left(1-M \xi_{x}\right)^{\xi_{y} / \xi_{x}}-\sigma_{x} M \\
& \approx-\sigma_{x} M \quad \downarrow-\infty .
\end{aligned}
$$

For $k_{x ; p}=\frac{1}{\xi_{x}}$,

$$
\begin{aligned}
S\left(\frac{1}{\xi_{x}}\right) & =\mu_{y}+\frac{\sigma_{y}}{\xi_{y}}\left[1-\left(1-\frac{1}{\xi_{x}} \xi_{x}\right)^{\xi_{y} / \xi_{x}}\right]-\mu_{x}-\sigma_{x} \frac{1}{\xi_{x}} \\
& \approx-\frac{\sigma_{y}}{\xi_{y}} 0^{\xi_{y} / \xi_{x}} \downarrow-\infty \text { as } \frac{\xi_{y}}{\xi_{x}}<0 .
\end{aligned}
$$

The condition for $S\left(k_{x ; p}\right)>0$ is not satisfied as $k_{x ; y} \rightarrow \infty$. 


\section{Some extensions and generalizations}

When $\xi_{x}=\xi_{y}=\xi$, we can combine and simplify Conditions 1-3 of Theorem 1, i.e. $\xi_{x}=$ $\xi_{y}>0, \xi_{x}=\xi_{y}<0$ and $\xi_{x}=\xi_{y}=0$. We see the importance of the sign of $\xi$ compared to the sign of $\sigma_{x}-\sigma_{y}$ leads to the definition and corollary as follows

Definition 1 The function sign( $x)$ is such that

$$
\operatorname{sign}(x)=\left\{\begin{array}{rll}
-1 & \text { for } & x<0 \\
1 & \text { for } & x>0 \\
0 & \text { for } & x=0
\end{array}\right.
$$

Corollary 1 Let $Z_{x} \sim \operatorname{GEVM}\left(\mu_{x}, \sigma_{x}, \xi_{x}\right)$ and $Z_{y} \sim \operatorname{GEVM}\left(\mu_{y}, \sigma_{y}, \xi_{y}\right)$ then suppose we assume that $\xi_{x}=\xi_{y}=\xi$ for the relationship $Z_{y}>Z_{x}$ to hold then if $\operatorname{sign}(\xi)=\operatorname{sign}\left(\sigma_{x}-\sigma_{y}\right)$, then $\mu_{y}>\mu_{x}+\eta\left(\sigma_{x}, \sigma_{y}, \xi\right)$ where

$$
\eta\left(\sigma_{x}, \sigma_{y}, \xi\right)=\frac{\sigma_{x}-\sigma_{y}}{\xi} \text { with } \eta(\sigma, \sigma, 0)=0 .
$$

Proof

From Theorem 1, by considering all $\xi_{x}=\xi_{y}=\xi$ cases. When $\xi>0$ and $\sigma_{x}>\sigma_{y}$ then $\operatorname{sign}(\xi)=1$ and $\operatorname{sign}\left(\sigma_{x}-\sigma_{y}\right)=1$ with $\frac{\sigma_{x}-\sigma_{y}}{\xi}=\eta\left(\sigma_{x}, \sigma_{y}, \xi\right)$. When $\xi<0$ and $\sigma_{x}<\sigma_{y}$ then $\operatorname{sign}(\xi)=-1$ and $\operatorname{sign}\left(\sigma_{x}-\sigma_{y}\right)=-1$ with $\frac{\sigma_{x}-\sigma_{y}}{\xi}=\eta\left(\sigma_{x}, \sigma_{y}, \xi\right)$. When $\xi=0$ and $\sigma_{x}=\sigma_{y}$ then $\operatorname{sign}(\xi)=0$ and $\operatorname{sign}\left(\sigma_{x}-\sigma_{y}\right)=0$ with $\frac{\sigma_{x}-\sigma_{y}}{\xi}=\eta\left(\sigma_{x}, \sigma_{y}, \xi\right)=0$ as $\sigma_{x}=\sigma_{y}=\sigma$ and $\xi=0$. Combining all the three cases then $Z_{y}>Z_{x}$ when for $\operatorname{sign}(\xi)=$ $\operatorname{sign}\left(\sigma_{x}-\sigma_{y}\right)$ and $\mu_{y}>\mu_{x}+\eta\left(\sigma_{x}, \sigma_{y}, \xi\right)$ holds.

For Condition 4 of Theorem $1, \xi_{y}<0<\xi_{x}$, Lemma 1 is written for only the case when we assume $\sigma_{x}=\sigma_{y}=\sigma$. From Lemma 1 when $\xi_{x} \rightarrow 0$ and $\xi_{y} \rightarrow 0$, then Condition 3 of Theorem 1 is preferable. Neither $\sigma_{x}<\sigma_{y}$ nor $\sigma_{x}>\sigma_{y}$ case is presented here as no easy closed form found.

Lemma 1 For $\xi_{y}<0<\xi_{x}$ then $\mu_{y}+\frac{\sigma_{y}}{\xi_{y}}-\mu_{x}-\frac{\sigma_{x}}{\xi_{x}}>\sigma_{x}^{\xi_{y} /\left(\xi_{y}-\xi_{x}\right)} \sigma_{y}^{-\xi_{x} /\left(\xi_{y}-\xi_{x}\right)}\left(\frac{1}{\xi_{x}}-\frac{1}{\xi_{y}}\right)$. If we assume $\sigma_{x}=\sigma_{x}=\sigma$ then $\mu_{y}-\mu_{x}>2 \sigma A$, where $A=\frac{1}{\xi_{x}}-\frac{1}{\xi_{y}}>0$.

Proof

From Theorem 1, $\mu_{y}+\frac{\sigma_{y}}{\xi_{y}}-\mu_{x}-\frac{\sigma_{x}}{\xi_{x}}>\sigma_{x}^{\xi_{y} /\left(\xi_{y}-\xi_{x}\right)} \sigma_{y}^{-\xi_{x} /\left(\xi_{y}-\xi_{x}\right)}\left(\frac{1}{\xi_{x}}-\frac{1}{\xi_{y}}\right)$.

Let $\sigma_{x}=\sigma_{y}=\sigma$ then,

$$
\begin{aligned}
\mu_{y}+\frac{\sigma}{\xi_{y}}-\mu_{x}-\frac{\sigma}{\xi_{x}} & >\sigma^{\xi_{y} /\left(\xi_{y}-\xi_{x}\right)} \sigma^{-\xi_{x} /\left(\xi_{y}-\xi_{x}\right)}\left(\frac{1}{\xi_{x}}-\frac{1}{\xi_{y}}\right) \\
\mu_{y}-\mu_{x}+\sigma\left(\frac{1}{\xi_{y}}-\frac{1}{\xi_{x}}\right) & >\sigma\left(\frac{1}{\xi_{x}}-\frac{1}{\xi_{y}}\right) \\
\mu_{y}-\mu_{x} & >2 \sigma\left(\frac{1}{\xi_{x}}-\frac{1}{\xi_{y}}\right) .
\end{aligned}
$$


For Condition 5 of Theorem 1, three assumptions possible for the scale parameters, i.e. $\sigma_{y}<\sigma_{x}, \sigma_{x}<\sigma_{y}$ and $\sigma_{x}=\sigma_{y}$, as in the following three lemmas.

Lemma 2 For $\xi_{x}=0, \xi_{y}<0$ then $\mu_{y}+\frac{\sigma_{y}}{\xi_{y}}-\mu_{x}-\frac{\sigma_{x}}{\xi_{y}}+\frac{\sigma_{x}}{\xi_{y}} \log \left(\frac{\sigma_{x}}{\sigma_{y}}\right)>0$. If we assume $\sigma_{y}(1+\delta)=\sigma_{x}, \delta>0$ then $\mu_{y}>\mu_{x}+C$ with $C=\frac{\sigma_{y}}{\xi_{y}}[(1+\delta) \log (1+\delta)-\delta]<0$.

Proof

$$
\text { From, } \begin{aligned}
0 & <\mu_{y}+\frac{\sigma_{y}}{\xi_{y}}-\mu_{x}-\frac{\sigma_{x}}{\xi_{y}}+\frac{\sigma_{x}}{\xi_{y}} \log \left(\frac{\sigma_{x}}{\sigma_{y}}\right) \\
& =\mu_{y}-\mu_{x}-\frac{1}{\xi_{y}}\left[-\sigma_{y}+\sigma_{x}+\sigma_{x} \log \left(\frac{\sigma_{y}}{\sigma_{x}}\right)\right] .
\end{aligned}
$$

Suppose $\sigma_{y}<\sigma_{x}$, if we let $\sigma_{y}(1+\delta)=\sigma_{x}$, with $\delta>0$, then

$$
\begin{aligned}
{\left[-\sigma_{y}+\sigma_{x}+\sigma_{x} \log \left(\frac{\sigma_{y}}{\sigma_{x}}\right)\right] } & =\sigma_{y} \delta-\sigma_{y}(1+\delta) \log (1+\delta) \\
& =\sigma_{y}[\delta-(1+\delta) \log (1+\delta)]<0 .
\end{aligned}
$$

As $\xi_{y}<0$, then $C=-\frac{1}{\xi_{y}}\left[-\sigma_{y}+\sigma_{x}+\sigma_{x} \log \left(\frac{\sigma_{y}}{\sigma_{x}}\right)\right]=\frac{\sigma_{y}}{\xi_{y}}[(1+\delta) \log (1+\delta)-\delta]<0$.

Lemma 3 For $\xi_{x}=0, \xi_{y}<0$ then $\mu_{y}+\frac{\sigma_{y}}{\xi_{y}}-\mu_{x}-\frac{\sigma_{x}}{\sigma_{y}}+\frac{\sigma_{x}}{\xi_{y}} \log \left(\frac{\sigma_{x}}{\sigma_{y}}\right)>0$. If we assume $\sigma_{y}=\sigma_{x}(1+\delta), \delta>0$ then $\mu_{y}>\mu_{x}+C$ with $C=\frac{\sigma_{y}}{\xi_{y}}[\delta-\log (1+\delta)]>0$.

Proof

From equation (14), suppose $\sigma_{y}>\sigma_{x}$, if we let $\sigma_{y}=\sigma_{x}(1+\delta)$, with $\delta>0$, then

$$
\begin{aligned}
{\left[-\sigma_{y}+\sigma_{x}+\sigma_{x} \log \left(\frac{\sigma_{y}}{\sigma_{x}}\right)\right] } & =-\sigma_{x} \delta+\sigma_{x} \log (1+\delta) \\
& =\sigma_{x}[\log (1+\delta)-\delta]>0 .
\end{aligned}
$$

As $\xi_{y}<0$, then $C=-\frac{1}{\xi_{y}}\left[-\sigma_{y}+\sigma_{x}+\sigma_{x} \log \left(\frac{\sigma_{y}}{\sigma_{x}}\right)\right]=\frac{\sigma_{y}}{\xi_{y}}[\delta-\log (1+\delta)]>0$.

Lemma 4 For $\xi_{x}=0, \xi_{y}<0$ then $\mu_{y}+\frac{\sigma_{y}}{\xi_{y}}-\mu_{x}-\frac{\sigma_{x}}{\sigma_{y}}+\frac{\sigma_{x}}{\xi_{y}} \log \left(\frac{\sigma_{x}}{\sigma_{y}}\right)>0$. If we assume $\sigma_{y}=\sigma_{x}=\sigma$ then $\mu_{y}-\mu_{x}>0$.

\section{Proof}

From equation (14) if we let $\sigma_{y}=\sigma_{x}=\sigma>0$, then

$$
\begin{aligned}
\mu_{y}+\frac{\sigma}{\xi_{y}}-\mu_{x}-\frac{\sigma}{\xi_{y}}+\frac{\sigma}{\xi_{y}} \log 1 & >0 \\
\mu_{y}-\mu_{x} & >0 .
\end{aligned}
$$




\section{Conclusion}

Theorem 1 tells us that if we know that the stochastic ordering constraint $Z_{x}<Z_{y}$ holds for GEVM distributed random variables $Z_{x}$ and $Z_{y}$. We can simplify the possible parameter space for the marginal parameters of $Z_{x}$ and $Z_{y}$ into five conditions. The sign of the shape parameter helps in simplying Theorem 1 leading to Corollary 1 and Lemmas 1-4.

\section{Acknowledgement}

This project is partially funded by Grant FRGS 02-1-15-1741FR.

\section{References}

[1] Nadarajah, S. Approximations for bivariate extreme values. Extremes. 2000. 3: 87-98.

[2] Khaledi, B. and Kochar, S. C. Stochastic Ordering among Order Statistics and Sample Spacings. Technical Report. Indian Statistical Institute. 2002.

[3] Nadarajah, S., Anderson, C. W. and Tawn, J. A. Ordered multivariate extremes. Journal of Royal Statistical Society. Series B (Statistical Methodology). 1998. 60: 473-496.

[4] Pickands, J. Statistical inference using extreme order statistics. The Annals of Statistics. 1975. 3: 119-131.

[5] Adam, M. B. and Tawn, J. A. Modification of Pickands' dependence function for ordered bivariate extreme distribution. Communications in Statistics: Theory and Methods. 2010. 40(9): 1687-1700. 